\title{
Molecular Hydrogen in the Circumstellar Shells of Carbon Stars
}

\author{
KENNETH HINKLE ${ }^{1}$ and JOHN KEADY ${ }^{2}$
}

${ }^{1}$ NOAO/Kitt Peak National Observatory, Tucson AZ, U.S.A.

${ }^{2}$ Los Alamos National Laboratory, Los Alamos NM, U.S.A.

The S-branch lines of molecular hydrogen were detected in $2.0-2.4 \mu \mathrm{m}$ carbon-star spectra by Johnson et al. (1983, ApJ, 270, L63). We subsequently detected strong S-branch lines in the $2 \mu \mathrm{m}$ infrared spectra of M-, S-, and Ctype long-period variables. In long-period variables, the $\mathrm{H}_{2}$ lines show very strong phase-dependent changes in velocity, strength, and line profile, with the $\mathrm{H}_{2}$ line strength greatest near minimum light. The strength of the $\mathrm{H}_{2}$ lines also depends on the visual amplitude of the variable. Analysis of the $\mathrm{H}_{2}$ line profiles shows that the $\mathrm{H}_{2}$ line is formed both in the photosphere and in an extended, non-photospheric (non-pulsating) region (or regions) of the stellar atmosphere.

In the case of the obscured carbon stars, the photospheric $2 \mu \mathrm{m}$ spectrum has been nearly completely filled in by thermal emission from circumstellar dust. Our modeling of the circumstellar envelope of IRC +10216 indicated that the expanding circumstellar $\mathrm{H}_{2} \mathrm{~S}(1)$ line should be detectable with a depth of a percent or so. We also felt that observations of this line would shed light on the origin of the non-photospheric $\mathrm{H}_{2}$ profiles seen in non-obscured stars. We undertook very high signal-to-noise $2 \mu \mathrm{m}$ spectroscopy of IRC +10216 to observe the $\mathrm{H}_{2} \mathrm{~S}(1)$ line. However, the observations reveal that at the few percent level, the photospheric spectrum of IRC +10216 is present in the $2.12 \mu \mathrm{m}$ spectrum. This greatly complicates the analysis. Limits on the massloss rate will be discussed. 